\title{
The therapeutic effects of curcumin and capsaicin against cyclophosphamide side effects on the uterus in rats $^{1}$
}

\author{
Ercan Yilmaz', Rauf Melekoglu", Osman Ciftci"', Sevil Eraslan"v , Asli Cetinv, Nese Basak ${ }^{\mathrm{vI}}$
}

'Associate Professor, Medicine Faculty, Inonu University, Department of Obstetrics and Gynecology, Malatya, Turkey. Manuscript writing.

"Assistant Professor, Medicine Faculty, Inonu University, Department of Obstetrics and Gynecology, Malatya, Turkey. Acquisition of data.

I'Full Professor, Medicine Faculty, Pamukkale University, Department of Medical Pharmacology, Denizli, Turkey. Analysis of data.

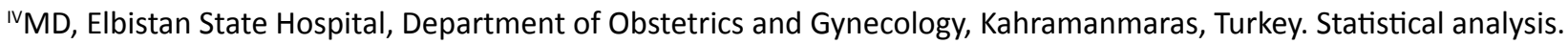

${ }^{\vee}$ Assistant Professor, Medicine Faculty, Inonu University, Department of Histology, Malatya, Turkey. Histopathological analysis.

V'MD, Pharmacy Faculty, Inonu University, Department of Pharmeceutical Toxicology, Malatya, Turkey. Acquisition of data.

\section{Abstract}

Purpose: To evaluate the impact of systemic cyclophosphamide treatment on the rat uterus and investigate the potential therapeutic effects of natural antioxidant preparations curcumin and capsaicin against cyclophosphamide side effects.

Methods: A 40 healthy adult female Wistar albino rats were used in this study. Rats were randomly divided into four groups to determine the effects of curcumin and capsaicin against Cyclophosphamide side effects on the uterus ( $n=10$ in each group); Group 1 was the control group (sham-operated), Group 2 was the cyclophosphamide group, Group 3 was the cyclophosphamide + curcumin $(100 \mathrm{mg} / \mathrm{kg})$ group, and Group 4 was the cyclophosphamide + capsaicin $(0.5 \mathrm{mg} / \mathrm{kg})$ group.

Results: Increased tissue oxidative stress and histological damage in the rat uterus were demonstrated due to the treatment of systemic cyclophosphamide chemotherapy alone. The level of tissue oxidant and antioxidant markers and histopathological changes were improved by the treatment of curcumin and capsaicin.

Conclusion: Cytotoxic effects of natural alkylating chemotherapeutic agents like cyclophosphamide on the uterus can be prevented by curcumin and capsaicin.

Key words: Cyclophosphamide. Curcumin. Capsaicin. Antioxidant. Rats. 


\section{- Introduction}

It is now accepted that the incidence of cancers of the organs and tissues has increased significantly in recent years throughout the world ${ }^{1}$. As is known, adjuvant and/or neoadjuvant chemotherapeutic agents have an essential role in addition to appropriate surgical treatment in cancer therapy. Besides the ability of chemotherapeutic agents to neutralize tumor cells, they also have significant adverse effects on many cells in the organism, especially those with anti-mitotic potency ${ }^{2}$.

Cyclophosphamide is an alkylating chemotherapeutic agent, and it has an extensive use for adjuvant or neoadjuvant purposes in the treatment of hematological malignancies, neuroblastoma, retinoblastoma, rhabdomyosarcoma, breast, lung, endometrium and ovarian cancers. Despite its frequent use, catastrophic side effects on the genitourinary, reproductive and gastrointestinal system organs are still not preventable today ${ }^{3}$. While the effect of $\mathrm{CYC}$ on ovary in the female genital system has been well described, there is limited data about the effect of CYC on the uterus.

The use of natural antioxidants in order to minimize the side effects of chemotherapeutic agents such as CYC has increased in recent years. Curcumin (CRC) and capsaicin (CPS) are the most prominent of these. CRC is derived from "Curcuma longa". Its homeland is East India and is defined as "the queen of spices". It includes natural polyphenol derivatives, gives a yellow color to natural turmeric plant, has anti-oxidant, anti-inflammatory, and apoptosis-inducing features and naturally inhibits cancer cell proliferation ${ }^{4}$. CPS is a trans-8-methyl-Nvanillyl-6-nonenamide compound obtained from chili pepper that is found in spices and topical medicines all over the world and has anti-inflammatory, antioxidant and antitumoral properties ${ }^{5}$.

Although the antitumoral properties of both compounds are known, how they prevent complex tumorigenic pathways characterized by uncontrolled cell proliferation, angiogenesis, invasion, and metastasis is still unclear. It is believed that this effect is achieved by inhibiting the transcription and growth factors, blocking the receptor, inactivating the kinase enzyme system, and inhibiting cytokine release which causes tumorigenesis. Similarly, the catastrophic effect of chemotherapeutic agents on the body is thought to be inhibited by the mechanisms outlined above ${ }^{6}$.

In this study, we aimed to show the beneficial effect of CRC and CPS treatment against the tissue damage due to systemic cyclophosphamide chemotherapy in rat uterus.

\section{- Methods}

The experiments were performed according to the animal ethics guidelines of the Inonu University Institutional Animal Ethics Committee (Ethical approval no: 2016/A-87).

A total of 40 healthy adult female Wistar albino rats (aged 3-4 months) obtained from The Experimental Animal Institute (Malatya, Turkey) were used in the experiment. Animals were housed in sterilized polypropylene rat cages under a 12/12-h light/dark cycle at an ambient temperature of $21^{\circ} \mathrm{C}$. Food and water were provided ad libitum. Rats were randomly divided into four groups to determine the effects of curcumin and capsaicin against cyclophosphamide side effects on the uterus ( $n=10$ in each group); Group 1 was the control group (sham-operated), Group 2 was the cyclophosphamide group, Group 3 was the cyclophosphamide + curcumin (100mg/ 
kg) $(C Y C+C R C)$ group, and Group 4 was the cyclophosphamide + capsaicin $(0.5 \mathrm{mg} / \mathrm{kg})$ (CYC+CPS) group. Chemotherapy induction was carried out by intraperitoneal injection of $200 \mathrm{mg} / \mathrm{kg}$ CYC (Endoxan, EIP ECZACIBASI, Istanbul, Turkey) on day 1 and then $8 \mathrm{mg} /$ $\mathrm{kg} /$ day for the following 14 days to the rats in group 2, 3, and 4 . After 14 days curcumin $(100 \mathrm{mg} / \mathrm{kg})$ and capsaicin $(0.5 \mathrm{mg} / \mathrm{kg})$ were orally administered for 14 days by gavages. The doses of CRC and CPS were chosen based on previous studies ${ }^{7,8}$. No treatment was given to the control group. The animals were sacrificed without pain or distress using increasing anaesthesia doses (intraperitoneal lethal doses of pentobarbital; Bioveta Inc., Ankara, Turkey) after the treatment course, and the uterine horns were removed for histopathological analyses. Blood samples were collected under anaesthesia from the left ventricle using a syringe. Tissue samples were stored at $-45^{\circ} \mathrm{C}$ until analysed.

\section{Biochemical assays}

Tissues were homogenized using a Teflon glass homogenizer in $150 \mathrm{mM}$ $\mathrm{KCl}(\mathrm{pH} 7.4)$ at a $1: 10(\mathrm{w} / \mathrm{v})$ dilution of the whole homogenate. The homogenates were centrifuged at $18.000 \times g$ and $4^{\circ} \mathrm{C}$ for $30 \mathrm{~min}$ to determine malondialdehyde (MDA) and reduced glutathione (GSH) concentrations and the superoxide dismutase (SOD) and catalase (CAT) activities or at $25.000 \times g$ for $50 \mathrm{~min}$ to determine the glutathione peroxidase (GPx) activity.

\section{Histopathological examinations}

For light microscopic evaluation, ovarian tissues were fixed in $10 \%$ formalin and embedded in paraffin wax. Paraffin waxembedded specimens were cut into five $\mu \mathrm{m}$ thick sections, mounted on slides, and stained with hematoxylin and eosin (H-E). The tissue samples were examined using a Leica DFC280 light microscope and a Leica Q-Win Image Analysis system (Leica Micros Imaging Solutions Ltd., Cambridge, UK). Histopathological examination of the tissue damage was performed regarding each parameter, such as inflammatory cells in the lumen, vascular congestion, epithelial degeneration and vacuoles in epithelial cells and dilated endometrial gland. At least five microscopic regions were examined to score the specimens semi-quantitatively. Each sample was scored for each criterion using a scale ranging from 0 to 3 ( 0 , none; 1 , mild; 2 , moderate; 3 , severe). Total scores were calculated according to these parameters.

\section{Statistical analysis}

All values were presented as the mean \pm standard deviation. Differences were considered significant at $p<0.01$. The SPSS software (ver. 18.0; SPSS Inc., Chicago, IL, USA) was used for statistical analyses. Biochemical values were analyzed using oneway ANOVA and post hoc Tukey's honestly significant difference test. Histological results were compared using Kruskal-Wallis variance analysis. When differences among the groups were detected, group means were compared using the Mann-Whitney U-test.

\section{Results}

\section{Biochemical results}

Antioxidant (SOD, CAT, GPx, and GSH) and oxidant parameters (MDA) in rat uterus are presented in Table 1. MDA levels were significantly decreased, whereas GSH levels, 
GPX, CAT, and SOD activities were significantly increased in uterine tissues in the CYC+CRC and the CYC+CPS groups compared with the control group.

Table 1 - MDA, GSH, SOD, GPx and CAT levels in tissues of the control group and the study group.

\begin{tabular}{llllll} 
Groups & MDA & GSH & SOD & GPX & CAT \\
\hline Control & $5.97 \pm 0.57^{\mathrm{a}}$ & $44.0 \pm 2.02^{\mathrm{a}}$ & $114.9 \pm 4.84^{\mathrm{a}}$ & $495.5 \pm 45.8^{\mathrm{a}}$ & $0.031 \pm 0.002^{\mathrm{a}}$ \\
CYC & $8.08 \pm 0.79^{\mathrm{b}}$ & $39.2 \pm 2.50^{\mathrm{b}}$ & $92.7 \pm 8.25^{\mathrm{b}}$ & $480.4 \pm 140.6^{\mathrm{b}}$ & $0.017 \pm 0.004^{\mathrm{b}}$ \\
CYC+CPS & $4.46 \pm 1.11^{\mathrm{c}}$ & $53.5 \pm 5.60^{\mathrm{c}}$ & $146.6 \pm 27.3^{\mathrm{c}}$ & $486.3 \pm 122.9^{\mathrm{c}}$ & $0.029 \pm 0.005^{\mathrm{c}}$ \\
CYC+CRC & $4.58 \pm 0.68^{\mathrm{c}}$ & $45.2 \pm 3.15^{\mathrm{ac}}$ & $123.3 \pm 28.9^{\mathrm{ac}}$ & $487.5 \pm 103.1^{\mathrm{c}}$ & $0.032 \pm 0.003^{\mathrm{ac}}$
\end{tabular}

*MDA: malonaldehyde, GSH: glutathione, SOD: superoxide dismutase, GPx: glutathione peroxidase, CAT: catalase, CYC: cyclophosphamide, CPS: Capsaicin, CRC: Curcumin.

$* * * *$ Mean values bearing different superscript letters within the same column are significantly different $(p<0.01)$.

Histological results

We observed some histopathological changes in uterine tissue in the CYC group. These histopathological changes were inflammatory cells in the lumen (Figure $1 \mathrm{~A}$ ), vascular congestion (Figure 1B), increased epithelial degeneration, vacuoles in epithelial cells (Figure 1C) and dilated endometrial glands (Figure 1D). Figure 2 shows little inflammatory cells in the lumen and normal endometrial gland. Improved epithelial degeneration and normal endometrial gland were shown in $A$ in $C Y C+C P S$ group. And $B$ shows few epithelial degeneration and vacuolization in epithelial cells in the $\mathrm{CYC}+\mathrm{CRC}$ group. Histopathologic scores for all four groups are demonstrated in Table 2. A significant increment in vascular congestion, inflammatory cells in the lumen, epithelial degeneration, vacuoles in epithelial cells and dilated endometrial gland were observed in the CYC group compared with the other groups. All histological parameters were significantly improved with the administration of CRC and CPS.

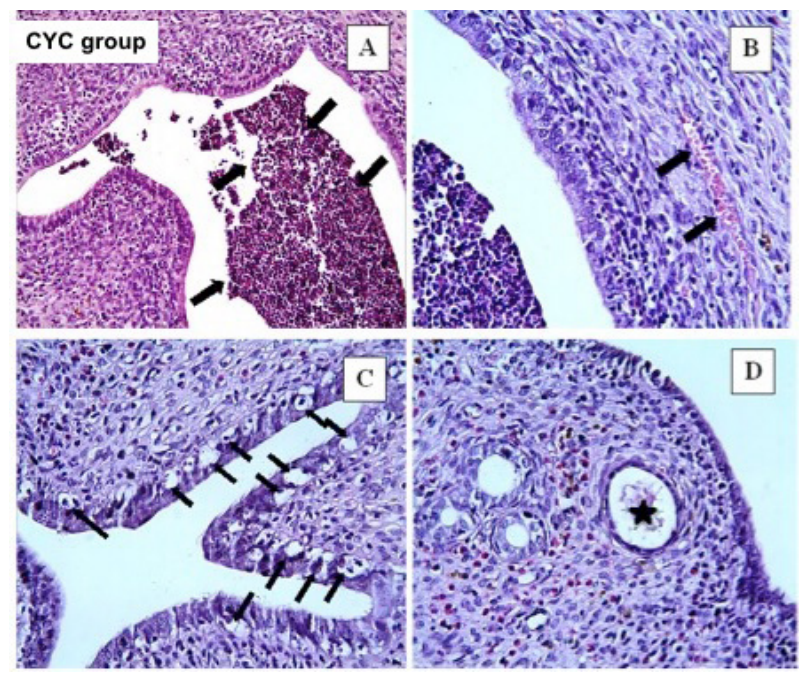

Figure 1 - Histopathological evaluation of rat uteruses given CYC. A: inflammatory cells were observed in lumen (black arrows), B: vascular congestion (black arrows), C: significantly increased epithelial degeneration and vocuolization in epithelial cells (black arrows), $\mathrm{D}$ : dilated endometrial gland (black asterix). A; H-E x20, B,C,D: H-E x40. CYC; cyclophosphamide, H-E; Hematoxylene-Eosine 


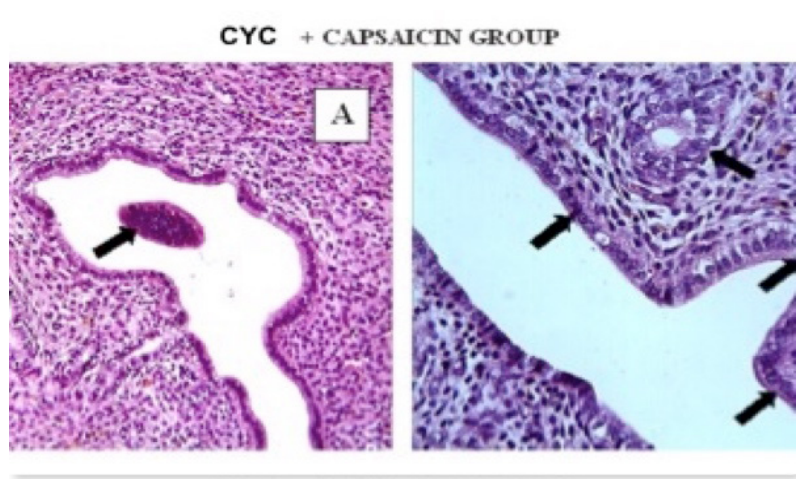

CYC + CURCUMIIN GROUP

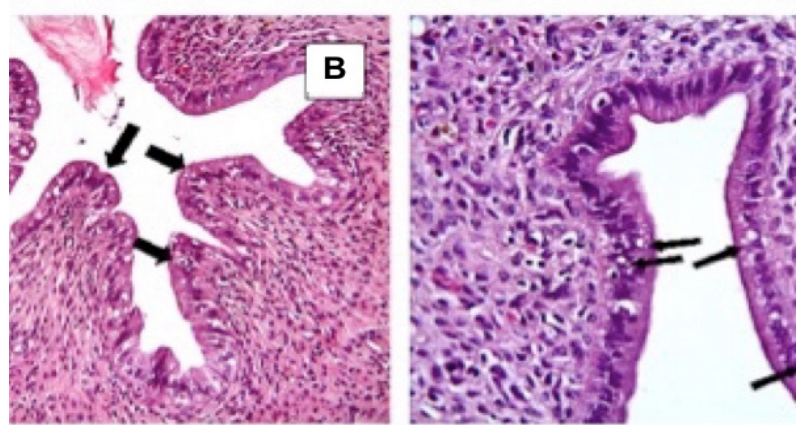

Figure 2 - Histopathological appearance of rat uteruses given $\mathrm{CYC}+\mathrm{CRC}$ and $\mathrm{CYC}+\mathrm{CPS}$. A: Significantly decreased inflammatory cell deposition, decreased epithelial degeneration and normal endometrial glandular tissue (black arrows) compared to the group given CYC, B: minimal epithelial degeneration, decreased epithelial vocuolization (black arrows), H-E x40. CYC; cyclophosphamide, CRC; curcumin, CPS; capsaicin, $\mathrm{H}-\mathrm{E}$; Hematoxylene-Eosine.

Table 2 - Histopathological score of groups.

\begin{tabular}{cc} 
Groups & $\begin{array}{c}\text { Histopathologic Damage } \\
\text { (Mean } \pm \text { SD) }\end{array}$ \\
\hline Control & $0.45 \pm 0.07^{\mathrm{a}}$ \\
CYC & $2.99 \pm 0.21^{\mathrm{b}}$ \\
CYC+ CPS & $1.35 \pm 0.25^{\mathrm{c}}$ \\
CYC + CRC & $1.28 \pm 0.10^{\mathrm{c}}$ \\
\hline
\end{tabular}

*CYC: cyclophosphamide; CRC: curcumin; CPS: capsaicin

** Mean values bearing different superscript letters within the same column are significantly different $(p<0.01)$.

\section{- Discussion}

Physicians are sometimes overwhelmed in the treatment of cancer due to adverse environmental factors despite technological advances. Adjuvant and/or neoadjuvant chemotherapeutic agents considered crucial in cancer treatment to enhance patient survival. Alkylating agents such as CYC inhibit DNA synthesis in tumor cells as well as have cytotoxic effects in the gastrointestinal system, liver and cardiac tissue cells, and especially in the genital system ${ }^{9}$. Although the effect of CYC on ovary in the female genital system has been well defined, the effect of CYC on the uterus has not been evaluated. To our knowledge, this is the first report to investigate the effects of CYC on the uterus, and this study demonstrated the detrimental effect of CYC on rat uterus by biochemical and histopathological analyses.

CYC, a compound of $\mathrm{N}, \mathrm{N}$-bis (2-chloroethyl) tetrahydro-2H-1,3,2oxazaphosphorin 2-amine 2-oxide, is widely used in the treatment of autoimmune diseases such as systemic lupus erythematosus and rheumatoid arthritis in addition to its important place in cancer treatment. It is primarily metabolized by cytochrome P450 enzyme system in the liver after systemic administration and is converted into two basic compounds as phosphoramide mustard and acrolein. Phosphoramide mustard is responsible for basic antineoplastic activity and acts as a DNA alkylating agent independent from the cell cycle, while acrolein is responsible from cytotoxic effects by causing the formation of reactive oxygen species (ROS) in the organism ${ }^{10}$. In normal cell cycle, endogenous ROS is produced at the stages of mitochondrial respiration, NADPH oxidase activity, and peroxisome $\beta$ - oxidation. Besides, ROS may be produced exogenously (ionizing or ultraviolet radiation, chemotherapeutic agents, and environmental stimuli) at a cellular level ${ }^{11}$. When produced at low concentrations these radicals are neutralized by natural antioxidant activity (such as GSH, SOD, GPx, CAT), 
whereas high ROS activity negatively affects intracellular homeostasis by causing cell cycle arrest, cell dysfunction and consequent DNA damage ${ }^{12}$. This is especially shown as the cause of chemotherapy-induced cytotoxicity. This situation has directed physicians to the use of natural antioxidants to prevent cytotoxicity, an important health problem associated with chemotherapy. The results of this study suggest that both curcumin and capsaicin have a protective effect against CYC-induced uterine damage. An animal study showed that selenium, which has natural antioxidant property, decreased total oxidant status (TOS) and increased total antioxidant status (TAS) in the kidney tissue of rats given $\mathrm{CYC}^{10}$. Another in vitro study with Carvacrol, a monoterpenic phenol compound with antioxidant activity found in aromatic plants such as thyme, showed that TOS and oxidative stress index decreased and antioxidant markers such as GSH, SOD, GPx, and CAT significantly increased in kidney tissues of CYC administered rats ${ }^{13}$.

Two important natural substrates used as antioxidants and also used in our study are CRC and CPS. CPS provides basic activity by pro-apoptotic action in the cell cycle. It inhibits the Nuclear factor-kappa beta pathway ${ }^{14}$, which plays an essential role in the aetiology of tumorigenesis and at the same time affects the cell cycle homeostasis negatively. It produces this effect indirectly by inhibiting the synthesis of transient receptor potential vanilloid receptors ${ }^{15}$. CRC produces antioxidant and anti-inflammatory activity by inhibiting the synthesis of the inducible nitric oxide synthase enzyme and inflammatory cytokines (such as interleukin-1 $\beta$ and 6 ). Also, CRC contributes to the inhibition of ROS by increasing activities of enzymes and genes such as NAD (P) $\mathrm{H}$, quinone oxidoreductase 1 and glutathione $\mathrm{S}$-transferase that are responsible for detoxification ${ }^{16}$. In vitro studies examining the antioxidant effect of CRC showed promising results. An in vitro rat study demonstrated that renal toxicity due to cyclosporine decreased significantly by CRC administration and TOS and MDA levels in this group were significantly low ${ }^{17}$. Another animal study detected that in renal tissues of cisplatin and methotrexate administered rats MDA level was increased and GSH level was decreased; however in CRC administered rats level of oxidant MDA significantly decreased and level of antioxidant GSH significantly increased ${ }^{18}$. Another in vitro study which evaluated cisplatin toxicity and CRC effectiveness in female rats in which experimental breast cancer was induced by 12-dimethyl benz[a]anthracene; TNF- $\alpha$, interleukin 6 and interleukin-8 activities significantly decreased in the group which was given cisplatin and CRC compared with the group which was given only CRC. The same study also evaluated the effect of CRC on cell homeostasis. Histopathological evaluation of breast tissue showed that cellular degeneration, necrosis, and hemorrhage were severe-very severe in the cisplatin administered group. However, none of these pathological findings occurred in the CRC+ cisplatin group ${ }^{19}$. Our study also showed that oxidant activity (MDA) was high and antioxidant activity (GSH, SOD, GPx, and CAT) was low in the uterus of rats treated with CYC; however in the uterus of the rats in the CRC+CYP group oxidant activity was low and antioxidant activity was high. In the histopathological examination, inflammation, congestion, epithelial degeneration and vacuolization were detected in the rat uterus exposed to CYC chemotherapy. However, in the CRC and CYP group, we found that these pathological findings were improved.

Although several studies showed cell protective effects of CPS due to its antioxidant and anti-inflammatory effects, there are 
limited studies to show the preventative effect of CPS against increased cytotoxicity due to chemotherapy. A study by Huang et al. ${ }^{20}$ showed the neuroprotective effect of CPS in cortical neurons of rats after ischemia/ reperfusion injury. In another study on rats, it has been demonstrated that the oxidative damage caused by alcohol in liver tissues of CPS-treated animals is minimized and that MDA and hydrogen peroxide-dependent oxidative processes are inhibited by CPSrelated antioxidant activity ${ }^{21}$. As mentioned, studies investigating CPS activity in order to prevent the increased cytotoxic effect due to chemotherapy are limited, but in an animal study performed Manjunatha et al. ${ }^{22}$ detected histopathological examination of the tissues showed a marked decrease in inflammatory cell accumulation and epithelial degeneration in the hepatic tissues of the group given CRC and CPC. In our study also showed decreased inflammation and prevention of tissue degeneration in histopathological evaluation of uterus tissues of rats given CPS.

Chemotherapeutic agents, which are indispensable for treatment in increasing cancer cases around the world, have positive effects on patient survival. Especially alkylating agents like CYC, prevents DNA synthesis in tumor cell not specific to mitosis stages and therefore blocks uncontrolled divisions of cancer cells. In addition to these effects, chemotherapeutic agents cause serious cytotoxic effects due to increased ROS, especially in liver, kidney and gastrointestinal system. These effects on the genital system have become a critical problem especially for chemotherapeutic agents applied in cancer cases diagnosed in younger ages in recent years. This caused focusing of physicians on specific antioxidant preparations especially natural ones. These antioxidant preparations also include CRC and CPS which are entirely natural. In our study, CPS and CRC were shown to have protective effects on the uterus in rats treated with CYC, taking both oxidant and antioxidant parameters into consideration. Besides, histopathological evaluations also confirmed these tissue protective effects of CRC and CPS. To the best of our knowledge, this study is the first one to demonstrate the cytotoxic effects of cyclophosphamide on the uterus in the female genital system, and also the results suggest that these cytotoxic effects can be treated by CRC and CPS. We believe that this study would have a guiding role for further studies in that it demonstrated that damage of chemotherapy on the genital system is reversible and preventable.

\section{- Conclusion}

Cytotoxic effects of natural alkylating chemotherapeutic agents like cyclophosphamide on the uterus can be prevented by curcumin and capsaicin

\section{References}

1. Yilmaz E, Coskun El, Sahin N, Ciplak B, Ekici K. MPV, NLR, and platelet count: new hematologic markers in diagnosis of malignant ovarian tumour. Eur J Gynaecol Oncol. 2017;38:346-9. PMID: 29693870.

2. Pyun CW, Kim JH, Han $\mathrm{KH}$, Hong GE, Lee $\mathrm{CH}$. In vivo protective effects of dietary curcumin and capsaicin against alcohol-induced oxidative stress. Biofactors. 2014;40:494500. doi: 10.1002/biof.1172.

3. Pascuali N, Scotti L, Di Pietro M, Oubiña G, Bas D, May M, Gómez Muñoz A, Cuasnicú PS, Cohen DJ, Tesone M, Abramovich D, Parborell F. Ceramide-1-phosphate has protective properties against cyclophosphamideinduced ovarian damage in a mice model of premature ovarian failure. Hum Reprod. 2018;33:844-59. doi: 10.1093/humrep/ dey045.

4. Kreutz $D$, Sinthuvanich $C$, Bileck $A$, Janker $L$, Muqaku B, Slany A, Gerner C. Curcumin exerts 
its antitumor effects in a context dependent fashion. J Proteomics. 2018;182:65-72. doi: 10.1016/j.jprot.2018.05.007.

5. El-Kott AF, Bin-Meferij MM. Suppressive effects of capsaicin against $\quad \mathrm{N}$-nitrosomethylurea-induced mammary tumorigenesis in rats. Biomed Pharmacother. 2018;98:673-9. doi: 10.1016/j.biopha.2017.12.036.

6. Wojcik M, Krawczyk M, Wojcik P, Cypryk K, Wozniak LA. Molecular mechanisms underlying curcumin-mediated therapeutic effects in type 2 diabetes and cancer. Oxid Med Cell Longev. 2018;20:1-14. doi: 10.1155/2018/9698258.

7. Ciftci O, Ozdemir I, Tanyildizi S, Yildiz $\mathrm{S}$, Oguzturk $\mathrm{H}$. Antioxidative effects of curcumin, $\beta$-myrcene and 1,8-cineole against $\quad 2,3,7,8$-tetrachlorodibenzo-pdioxin-induced oxidative stress in rats liver. Toxicol Ind Health. 2011;27:447-53. doi: $10.1177 / 0748233710388452$.

8. Huang $M$, Cheng $G$, Tan $H$, Qin R, Zou $Y$, Wang $Y$, Zhang Y. Capsaicin protects cortical neurons against ischemia/reperfusion injury via down-regulating NMDA receptors. Exp Neurol. 2017;295:66-76. doi: 10.1016/j. expneurol.2017.05.001.

9. Kurauchi K, Nishikawa T, Miyahara E, Okamoto Y, Kawano Y. Role of metabolites of cyclophosphamide in cardiotoxicity. BMC Res Notes. 2017;10:1-10. doi: 10.1186/ s13104-017-2726-2.

10.Gunes S, Sahinturk V, Uslu S, Ayhanci A, Kacar S, Uyar R. Protective effects of selenium on cyclophosphamide-induced oxidative stress and kidney injury. Biol Trace Elem Res. 2017;30:1-8. doi: 10.1007/ s12011-017-1231-8.

11.Wang GF, Dong Q, Bai Y, Yuan J, Xu Q, Cao $C$, Liu $X$. Oxidative stress induces mitotic arrest by inhibiting Aurora A-involved mitotic spindle formation. Free Radic Biol Med. 2017;103:177-87. doi: 10.1016/j. freeradbiomed.2016.12.031.

12.Turhan U, Yilmaz E, Gul M, Melekoglu R, Turkoz Y, Ozyalin F, Parlakpinar $H$, Simsek $Y$. Investigation of the effect of gestational diabetes on fetal cardiac tissue in streptozotocin induced in rats. Acta Cir Bras. 2018;33:306-13. doi: 10.1590/s0102865020180040000002 .
13.Gunes S, Ayhanci A, Sahinturk V, Altay DU, Uyar R. Carvacrol attenuates cyclophosphamide-induced oxidative stress in rat kidney. Can J Physiol Pharmacol. 2017;95:844-9. doi: 10.1139/cjpp-20160450.

14.Yilmaz E, Coskun El, Gul M, Sahin N, Tuncay G, Simsek Y. Nuclear factor- k a p p a beta pathway and endometrial cancer: a pilot study. Eur J Gynecol Oncol. 2017;38:536-40. doi: 10.12892/ejgo3471.2017.

15. Melekoglu R, Ciftci O, Eraslan S, Cetin A, Basak N. Beneficial effects of curcumin and capsaicin on cyclophosphamide-induced premature ovarian failure in a rat model. J Ovarian Res. 2018;11:33:1-8. doi: 10.1186/ s13048-018-0409-9.

16.Jaja-Chimedza A, Graf BL, Simmler C, Kim Y, Kuhn P, Pauli GF, Raskin I. Biochemical characterization and anti-inflammatory properties of an isothiocyanate-enriched moringa (Moringa oleifera) seed extract. PLoS One. 2017;12:1-21. doi: 10.1371/ journal.pone.0182658.

17. Huang J, Yao X, Weng G, Qi H, Ye X. Protective effect of curcumin against cyclosporine A-induced rat nephrotoxicity. Mol Med Rep. 2018;17:6038-44. doi: 10.3892/ mmr.2018.8591.

18.Said Salem NI, Noshy MM, Said AA. Modulatory effect of curcumin against genotoxicity and oxidative stress induced by cisplatin and methotrexate in male mice. Food Chem Toxicol. 2017;105:370-6. doi: 10.1016/j.fct.2017.04.007.

19.Kumar P, Barua CC, Sulakhiya K, Sharma RK. Curcumin ameliorates cisplatininduced nephrotoxicity and potentiates its anticancer activity in sd rats: potential role of curcumin in breast cancer chemotherapy. Front Pharmacol. 2017;8:1-12. doi: 10.3389/ fphar.2017.00132.

20. Huang $M$, Cheng G, Tan H, Qin R, Zou Y, Wang $Y$, Zhang Y. Capsaicin protects cortical neurons against ischemia/reperfusion injury via down-regulating NMDA receptors. Exp Neurol. 2017;295:66-76. doi: 10.1016/j. expneurol.2017.05.001.

21.Pyun CW, Kim JH, Han KH, Hong GE, Lee CH. In vivo protective effects of dietary curcumin and capsaicin against alcohol-induced oxidative stress. Biofactors. 2014;40:494- 
500. doi: 10.1002/biof.1172.

22. Manjunatha $H$, Srinivasan K. Protective effect of dietary curcumin and capsaicin on induced oxidation of low-density lipoprotein, iron-induced hepatotoxicity and carrageenan-induced inflammation in experimental rats. FEBS J. 2006;273:452837. PMID: 16956363.

\section{Correspondence:}

Ercan Yilmaz

Inonu University, Medicine Faculty,

Obstetrics and Gynecology Department

Malatya Turkey

Phone: +0905369556180

ercanyilmazgyn@yahoo.com

Received: Feb 22, 2018

Review: Apr 25, 2018

Accepted: May 23, 2018
Conflict of interest: none

Financial source: none
${ }^{1}$ Research performed at Medicine Faculty, Inonu University, Histology and Embryology and Animal Study, Malatya, Turkey. 